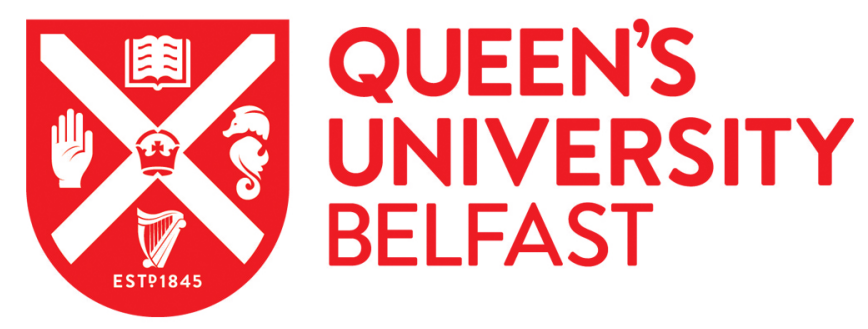

\title{
Intrinsic Controllable Magnetism of Graphene Grown on Fe
}

Hong, J., Kim, Y., Liang, J., Chen, H., Park, C. Y., Yang, H., Santos, E. J. G., Bokor, J., Hwang, C. C., \& You, L. (2019). Intrinsic Controllable Magnetism of Graphene Grown on Fe. Journal of Physical Chemistry C, 123(44), 26870-26876. https://doi.org/10.1021/acs.jpcc.9b05886

Published in:

Journal of Physical Chemistry C

Document Version:

Peer reviewed version

Queen's University Belfast - Research Portal:

Link to publication record in Queen's University Belfast Research Portal

\section{Publisher rights}

(C) 2019 American Chemical Society. This work is made available online in accordance with the publisher's policies. Please refer to any applicable terms of use of the publisher.

\section{General rights}

Copyright for the publications made accessible via the Queen's University Belfast Research Portal is retained by the author(s) and / or other copyright owners and it is a condition of accessing these publications that users recognise and abide by the legal requirements associated with these rights.

Take down policy

The Research Portal is Queen's institutional repository that provides access to Queen's research output. Every effort has been made to ensure that content in the Research Portal does not infringe any person's rights, or applicable UK laws. If you discover content in the Research Portal that you believe breaches copyright or violates any law, please contact openaccess@qub.ac.uk. 


\section{Intrinsic controllable magnetism of graphene grown on $\mathrm{Fe}$}

Jeongmin Hong, ${ }^{1 *}$ Yooseok Kim, ${ }^{2}$ Jinghua Liang, ${ }^{3}$ Hong Chen, ${ }^{4}$ Gong Chen, ${ }^{5}$ Andreas Schmid, Chong-Yun Park, ${ }^{2}$ Hong-Xin Yang, ${ }^{3}$ Elton J. G. Santos, ${ }^{6}$ Jeffrey Bokor, ${ }^{7}$ Chan-Cuk Hwang, ${ }^{8 *}$ Long You ${ }^{*}$

${ }^{1}$ School of Optical and Electronic Information, Huazhong University of Science and Technology, Wuhan, Hubei 430037, P.R. China.

${ }^{2}$ Department of Physics, Sungkyunkwan University, Suwon 16419, Korea (Republic of).

${ }^{3}$ Key Laboratory of Magnetic Materials and Devices, Ningbo Institute of Materials Technology and Engineering, Chinese Academy of Sciences, Ningbo 315201, P. R. China.

${ }^{4}$ School of Materials Science and Energy Engineering, Foshan University, Foshan, Guangdong 528000, P.R. China.

${ }^{5}$ The Molecular Foundry, Lawrence Berkeley National Laboratory, Berkeley, CA 94720, USA.

${ }^{6}$ School of Mathematics and Physics, Queen's University Belfast, BT7 1NN, UK, and

Department of Chemical Engineering, Stanford University, Stanford, CA 94305, USA.

${ }^{7}$ EECS, UC Berkeley, Berkeley, CA 94720, USA.

${ }^{8}$ Beamline division, Pohang Accelerator Laboratory (PAL), POSTECH, Pohang 37673, Korea

17 (Republic of).

\section{*Correspondence should be addressed to JH (jehong@hust.edu.cn); $\mathrm{CCH}$} (cchwang@postech.ac.kr); LY (lyou@hust.edu.cn)

Novel functional materials that use the spin and charge of the electron together, offer many exciting opportunities for the creation of new information processing and storage devices with ultralow power consumption. Recent discovery of magnetism in atomically thin layered materials could boost the research field of light element materials such as van der Waals magnetic nanostructures. Here, we report an intrinsic magnetization in large area graphene grown on $\mathrm{Fe}(100)$ foil structures. The optimal growing mechanisms provide graphene samples with high-quality and with controllable magnetic properties inducing thickness-dependent magnetization. Using first-principles calculations, we investigated several possible scenarios for controlling magnetic properties. Moreover, independent magnetic structures induced in graphene are identified with field-applied magnetic force microscopy (FA-MFM) followed by vibrating sample magnetometry (VSM) magnetometry. Our results open a new avenue for controllable magnetic properties of graphene structures mediated by surface growth on regular magnets for applications at large scale spintronics.

Computing using magnetic chips can reduce power consumption to one millionth of the amount of energy used by today's transistors. ${ }^{1-3}$ Such materials could be designed with multifunctional characteristics including controllable magnetic properties and superior electrical, photonic, thermal, and many other physical properties. The quantum phenomena in magnetic graphene include quantum spin hall effects, long spin-relaxation times, a long electronic mean free path, and ballistic spin transport at room temperature with the possibility to tune spin-orbit 
interactions..$^{4-8}$ Moreover, spin-diffusion length of graphene has been reported to $1 \sim 2 \mu \mathrm{m}$ at room temperature. ${ }^{7}$ Recently developed van der Waals $2 \mathrm{D}$ magnetism called back the study of magnetic graphene. ${ }^{9}$ The multifunctional carbon-based 2D nanomaterials would perform information processing using spin states at Fermi level. The magnetic ordering and induced ferromagnetism of carbon-based nanostructures has long been investigated in a variety of ways. ${ }^{10-19}$ However, due to the lack of controllability and the non-uniformity of the synthesized structures, such magnetic carbon properties have struggled to be applicable in practical spintronic devices. For this reason, large area magnetic graphene has been required for the further practical applications.

49 Graphene was grown on 100- $\mu m$-thick $\mathrm{Fe}$ foils in a hot furnace. By varying deposition parameters such as time and temperature, the thickness of graphene has been systematically controlled. Fig 1 shows a schematic of synthesis (A), experimental setup (B), and a graph of synthesis conditions (C), respectively. As shown in Fig 1(A), the well-known growth mechanisms are described: surface adsorption, diffusion, and precipitation. ${ }^{20}$ Two mechanisms have been considered to govern the growth of graphene on typical catalyst such as $\mathrm{Cu}$ and $\mathrm{Ni}$, respectively. ${ }^{21,22}$ The extremely low carbon solubility of $\mathrm{Cu}$ leads to self-limited growth of graphene via simple thermal decomposition of hydrocarbons on the $\mathrm{Cu}$ surface as shown in scenario (I). In contrast, the high carbon solubility of $\mathrm{Ni}$ causes carbon to dissolve into the bulk, and graphene subsequently grows through surface segregation of carbons upon cooling from a metastable carbon-metal solid solution (II). The formation of graphene on Fe follows both I and II mechanisms, simultaneously. These experimental results show that graphene can also be synthesized by intrinsic carbons contained inside the iron substrates (III), which could be a unique mechanism for growing magnetic carbon. ${ }^{23}$ The detailed synthesis conditions and experimental parameters are shown in Materials and Experimental sections. We developed such a combined synthesis technique to achieve high-quality graphene on Fe by using Acetylene $\left(\mathrm{C}_{2} \mathrm{H}_{2}\right)$ feedstock even if $\mathrm{Fe}$ could dissolve carbon drastically and it is very challenging to grow bulk graphene onto Fe substrate. Layer-controlled graphene films were successfully synthesized on the substrate in selective growth windows with a finely tuned $\mathrm{C}_{2} \mathrm{H}_{2}, \mathrm{Ar}$, and $\mathrm{H}_{2}$ gas ratio and growth time which makes it possible to prove thickness-dependent magnetization as shown in Fig $1(\mathrm{C})$.

Fig 1(B) shows a schematic of CVD systems with graphene sample for the optimal synthesis. 30 min of pre-annealing and plasma sputtering to clean the surface was performed as shown in Fig 1(C). During pre-annealing process, Fe-based oxides and impurities onto the $\mathrm{Fe}$ surface could be removed. By controlling several parameters such as temperature ranges, gas composition, and flow rates, graphene onto $\mathrm{Fe}$ could be synthesized in a layer controllable way by changing the synthesis time as shown in Fig 1(C). The quality of the samples was monitored using highresolution photoemission spectroscopy (HRPES) at the 10D beamline of the Pohang accelerator laboratory (PAL) and commercially available x-ray photoelectron spectroscopy (XPS). Sharp C $1 \mathrm{~s}$ core level spectra indicate the graphene grown on $\mathrm{Fe}$ are comparable with those on $\mathrm{Ni}$ and $\mathrm{Cu}$ in quality. ${ }^{23,24}$ 
(A)

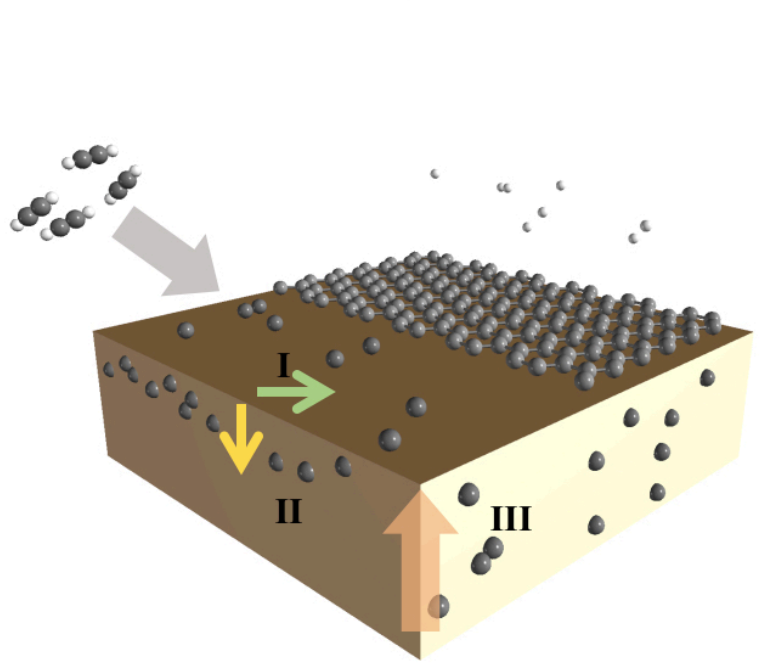

(B)

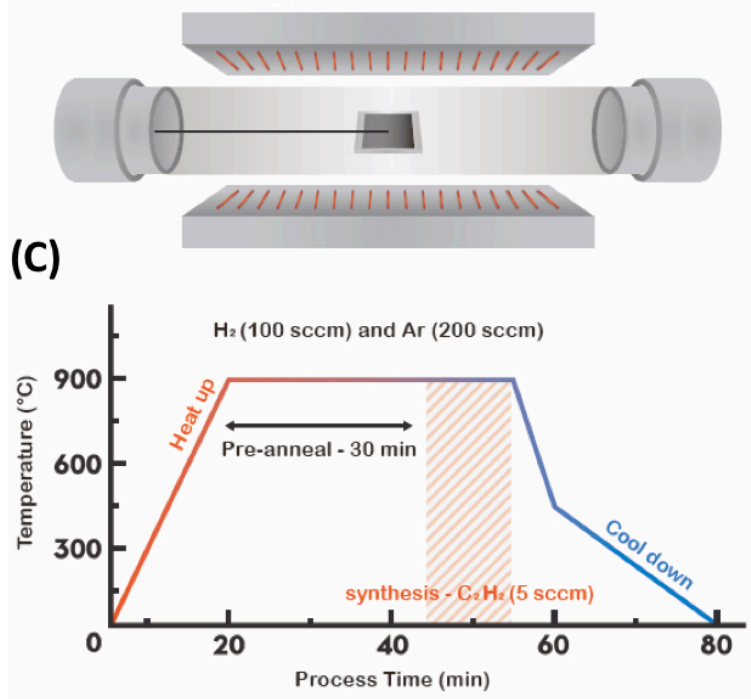

Figure 1 | Synthesis mechanisms of graphene on Fe: (A) Schematic of synthesis scenarios of magnetic graphene. (B) Schematic illustration of graphene growth in chemical vapor deposition (CVD) system. (C) Optimal parameters to synthesize graphene on Fe using CVD system.

In order to inspect the quality and exact thickness of the synthesized graphene, scanning electron microscopy (SEM) and high-resolution transmission electron microscopy (HR-TEM) measurements were performed for estimating the number of layers of graphene as shown in Figs 2(A)- $(\mathrm{H})$. The wrinkles are well known phenomena with the difference in thermal expansion coefficient between $\mathrm{Fe}$ and graphene when the foil was rapidly quenched or generated when a growing flake starts to overlap with another on nearby. The defects and edges are observed in multi-layers graphene films. Detailed synthesis, characterization process and the following results can be found in Materials and Methods section. The number of graphene layers can be clearly identified as $2,8,10$, and 13 layers, respectively, by virtue of the interlayer spacing in graphene $(0.34 \mathrm{~nm})$. As also shown in our previous reports, the amount of synthesis time could precisely control the number of layers. ${ }^{23-26}$

Raman spectroscopy is a powerful, yet relatively specimen-specific measurement to characterize the crystalline quality and number of graphene layers as shown in Fig 2(I). The D-peak depends on the breathing mode of the 6-fold aromatic ring $\left(\mathrm{sp}^{3}\right)$, which is independent of the number of graphene layers and is activated by disorder. The G-peak is due to the doubly degenerate zone in the center $E_{2 g}$ mode. Since generation of the 2D-peak is a process allowing symmetry, the band of the second order Raman process appears with no relation to any defect or any disorder. The 2D-peak even provides a count of the number of graphene sheets in the specimen. Other criteria used to measure the number of graphene layers are full width at half maximum (FWHM) and the position of the 2D-peak. Also, the intensity ratio of the $2 \mathrm{D}$ to G-peak $\left(I_{2 D} / I_{G}\right)$ is dependent on the number of layers.

As shown in Fig 2(J), the ratio of $I_{2 D} / I_{G}$ was around 1.61, 1.24, 0.75, and 0.55 corresponding to 1 , 3,6 , and $9 \mathrm{~min}$ of the synthesis time, respectively. In the bottom spectrum in Fig 2(J), the 
109 intensity ratio is 1.61 and the peak position of the symmetric 2D-band is $2700 \mathrm{~cm}^{-1}$ with a 110 FWHM of $35.5 \mathrm{~cm}^{-1}$ which is in accordance with the parameters for bilayer graphene.

111 Raman spectra are sometimes ambiguous in estimating the number of layers of graphene, 112 especially in few-layer graphene. The HR-TEM results agreed well with the Raman results and 113 clearly showed that the number of graphene layers increased when increasing the time of 114 synthesis. As a result, Raman spectra together with HR-TEM and SEM confirm different 115 numbers of layers and defects of the samples.
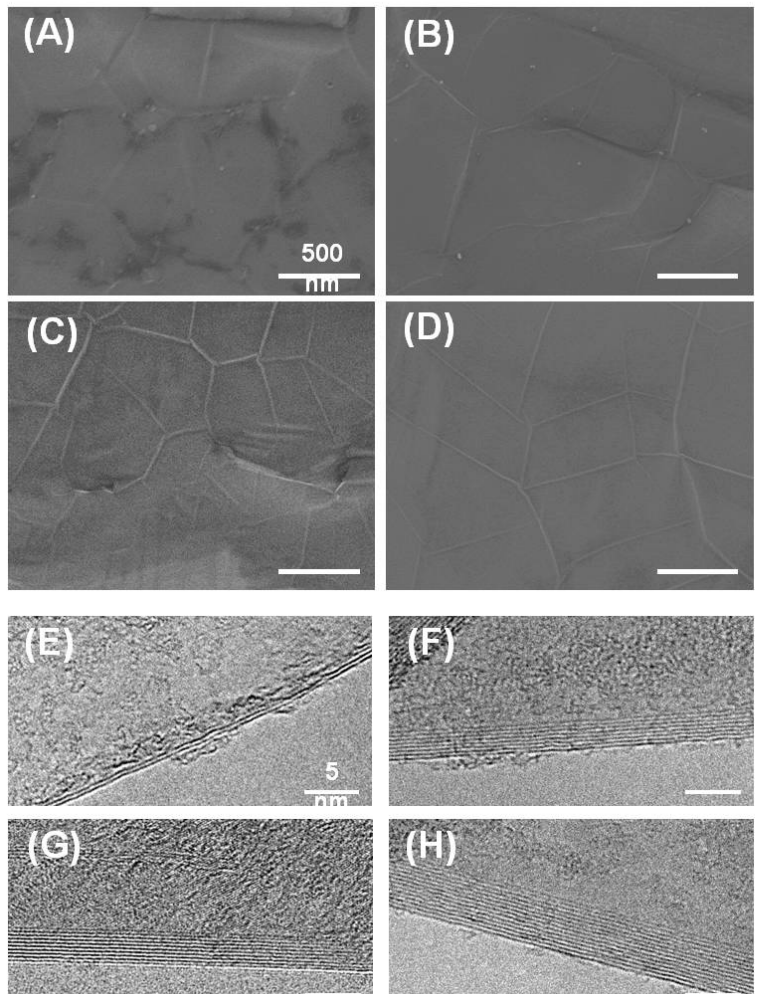
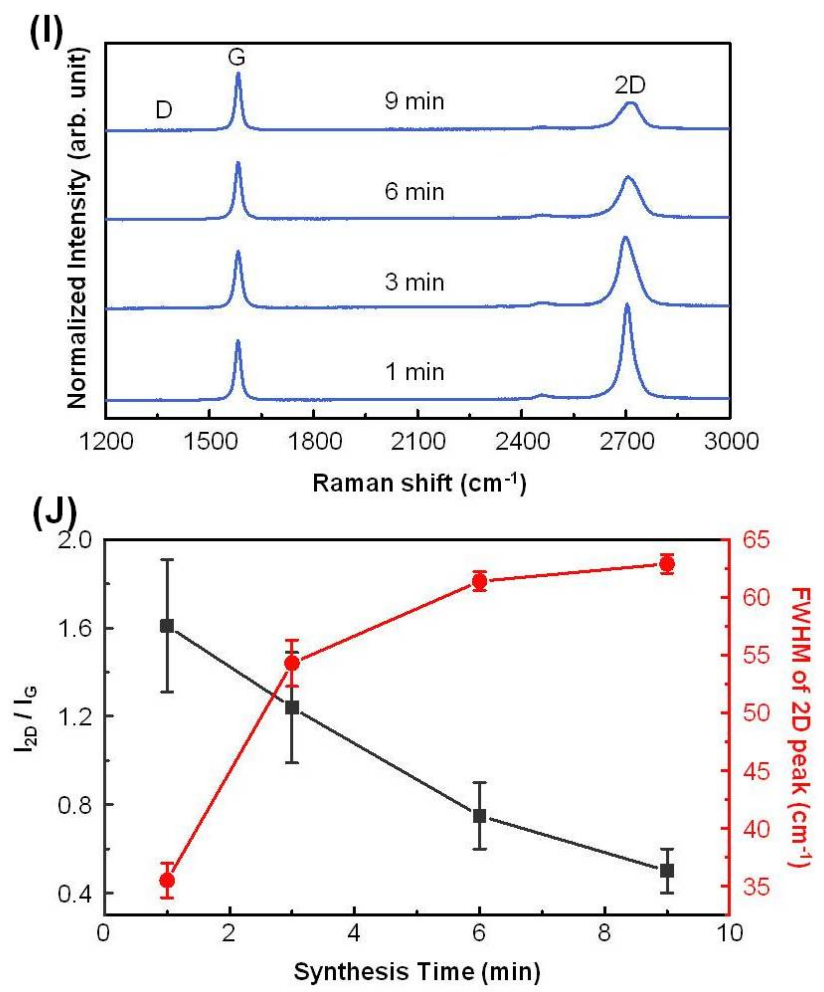

Figure 2 | The SEM micrographs of graphene by increasing the synthesis time from (A) 1 min, (B) $3 \mathrm{~min}$, (C) $6 \mathrm{~min}$, to (D) $9 \mathrm{~min}$. The TEM images during the synthesis: (E) 1 min (2 layers), (F) 3 min (8 layers), (G) 6 min (10 layers), and (H) 9 min (13 layers), respectively. Estimated thickness of the sample is as follows: 1 min of synthesis time for 2 layers, 3 min for 8 layers, $6 \mathrm{~min}$ for 10 layers, and $9 \mathrm{~min}$ for 13 layers, respectively. (I) The Raman spectra by increasing the synthesis time: $0 \mathrm{~min}, 1 \mathrm{~min}, 3 \mathrm{~min}, 6 \mathrm{~min}$, and $9 \mathrm{~min}$. (J) Ratio of 2D and G peaks and FWHM of 2D peaks. The estimation is based on Raman spectra and TEM.

Our study of Field-applied (FA)-MFM provides strong confirmation to probe the magnetic domain structure of materials in local regions. The FA-MFM image shown in Fig 3(A) indicates similar domain patterns of graphene grown on Fe foil which is very different from that of bcc $\mathrm{Fe}$ (100) bulk structures. The domain structures of Fe crystal will be changed from randomly oriented large domain to the smaller domain pattern after the synthesis of graphene. The magnetic stripe-domain structures that could be an indication of the majority of the crystalline 
structure after the synthesis process. As shown in Figs 3(A)-(D), the small domain structures are observed in graphene on Fe sample. The orientation of magnetization in the plane is shown in SI. The domain structures are changing by the increase of the layer. The results confirm controllable magnetic properties are observed in the domain patterns. The image contains topographical information and magnetic phases together because of the nature of graphene. However, the FAMFM could observe the phase change from fully saturated magnet underneath. Thus, the systems probe magnetic properties of carbon which indicate the independent domain formations from $\mathrm{Fe}$ substrates simultaneously.

141 After we transfer the sample to non-magnetic substrates, we can still observe small magnetization which confirm the effects are intrinsic. Using a stamping method, we could selectively transfer graphene on $\mathrm{Fe}$ to $\mathrm{SiO}_{2}$ substrates. Then, we carefully checked by x-ray spectra using synchrotron radiation at ALS. This indicates the aligned carbon edges and defects play a critical role for increasing magnetic moment in graphene. As shown in Fig 3(E), m-H loops indicate the presence of ferromagnetism (FM) and antiferromagnetism (AFM) together which already reported previously. ${ }^{13}$ Another important note is that light element magnetism usually comes from proximity effects which resulted from the hybridization between the transition metal and graphene. This is partly true, but the contribution could be smaller than the case of $\mathrm{Fe}$ foil because of the lattice mismatch. If we manipulate the systematic controllability, 151 spin devices could be built.
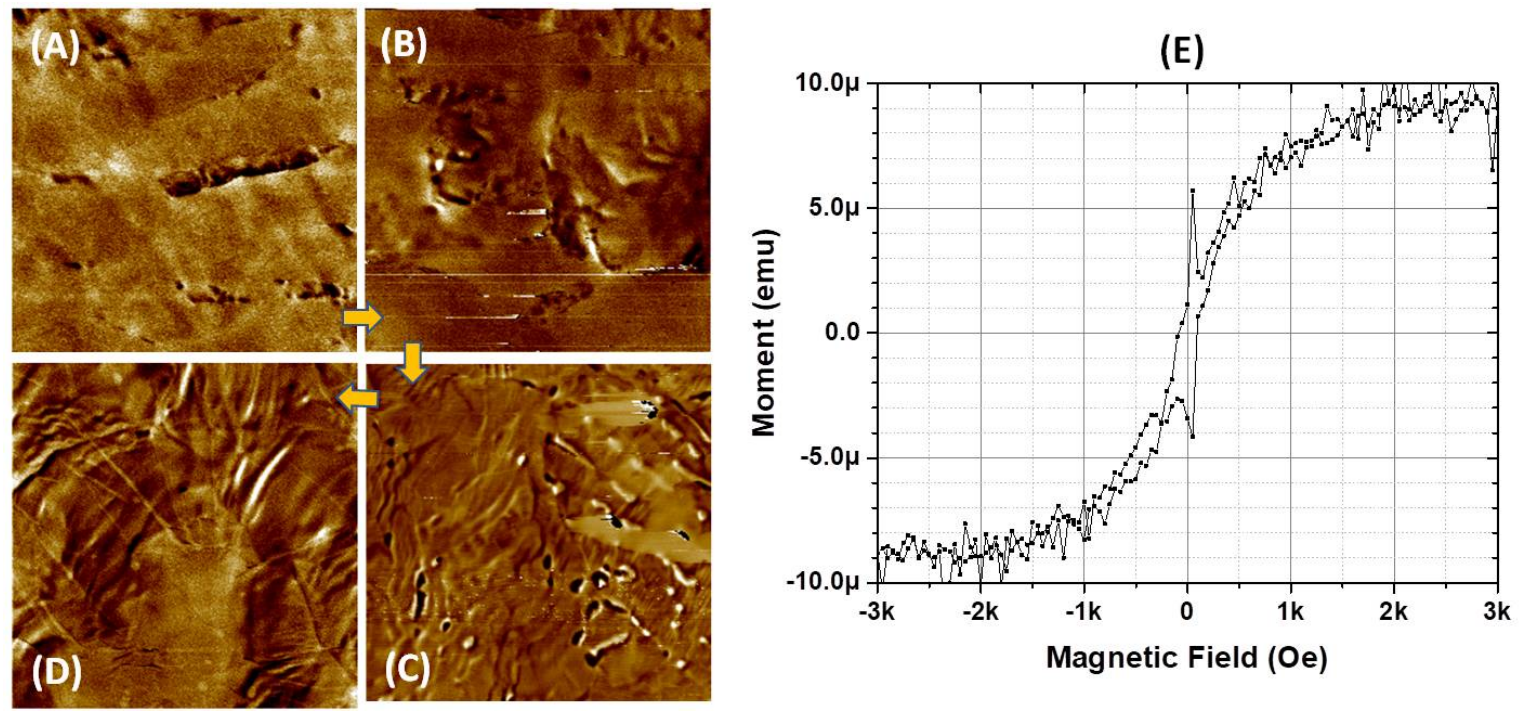

154 Figure 3 | Magnetic imaging characteristics. (A) Magnetic force microscopy (MFM) image of magnetic domains of graphene on Fe (100) foil which shows the different domain structures of $\mathrm{Fe}(100)$. The MFM images are observed by increasing the thickness: (A) 2, (B) 8 , (C) 10, (D) 13, respectively. The brighter and darker regions represent the change of the magnetic phase. The images are $3 \times 3 \mu \mathrm{m}^{2}$. (E) $\mathrm{m}$-H loop of graphene after transfer to $\mathrm{SiO}_{2}$ substrates. The presence of mixed antiferromagnetic (AFM) and ferromagnetic (FM) was observed. 
We turn on theoretical validation of the experimental results. The model structure of graphene grown on $\mathrm{Fe}$ is shown in Fig 4(A), where the spin polarized interface is highlighted. Once graphene is formed at the Fe surface, the proximity effect polarizes the carbon states near the Fermi level which just one spin channel is observed pinned at the Fermi level (Fig 4(B)). The Fe surface is the main sources of spin-polarized carriers as the amount of states per eV for one spin channel, e.g. spin-down, is substantially higher than that on spin-up (Figures S3(A) in Supporting Information). We also observed that the number of polarized states at graphene increases with the layer thickness, with a transition from one spin state to another as we crossed from monolayer (1L) to bi-layer (2L), that is, from spin up to spin down. The strong interactions between the nearest graphene layer and the $\mathrm{Fe}$ substrates make the exchange interactions favorable to this antiferromagnetic coupling by $15.4 \mathrm{meV}$ (Fig S3(B)). Fig 1(C) shows the magnetization at the first layers is also smaller than those at thicker systems, which can also be observed locally at each individual $\mathrm{C}$ atom at each layer (Fig 1(D)). This indicates an enhancement of the magnetic properties with the growth process, which incidentally saturates at about 15 carbon sheets on $\mathrm{Fe}$ as the increment in the magnetic moments begins decreasing thereafter. One of the main reasons associated with this thickness dependence can be observed on the amount of charge transfer from Fe substrates to graphene (Fig S3(C)), which is substantially larger for the layers near Fe and decays exponentially throughout the system. The amount of charge transfer is closely related to the position of the Fermi level at the interface which shows an oscillatory-like behavior with the layer thickness (Figs S3(D)), till it reaches a constant value around 9 layers. Then, more layers are deposited, the magnetic moment decays.

183 Fig 4(B) shows the magnetization at the first layers is also smaller than those at thicker systems, 184 which can also be observed locally at each individual carbon atom from each layer. This indicates an enhancement of the magnetic properties with the growth process, which incidentally saturates at about 15 complete carbon sheets on $\mathrm{Fe}$ as the increment in the magnetic moments 187 begins decreasing thereafter. Four possible cases are calculated for ferromagnetism: defects, 188 layer dependence, proximity effects, Fe intercalation as shown in Figure 4(C). Ideal defected and/or edge states in graphene show $0.4 \mathrm{Bohr}$, but in the experiment, it is estimated to $0.46 \mathrm{Bohr}$ from magnetization. One of the main reasons associated with this thickness dependence can be observed on the number of defects to graphene, which is substantially larger for the layers near $192 \mathrm{Fe}$ and decays exponentially throughout the system. Then, more layers are deposited, the magnetization decays that is spin density has been canted in thicker carbon. 
(A)

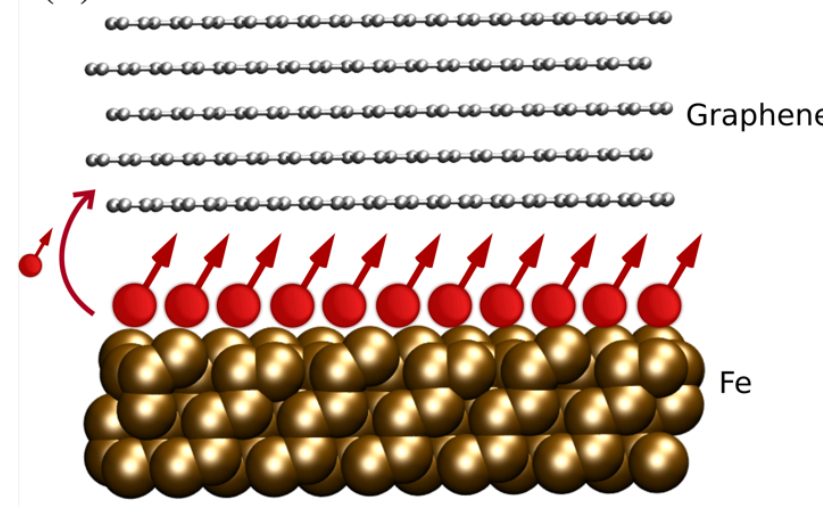

(B)

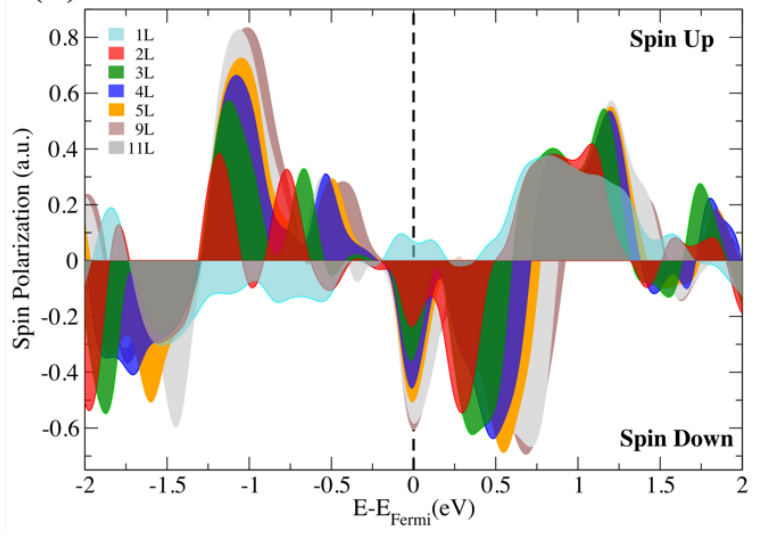

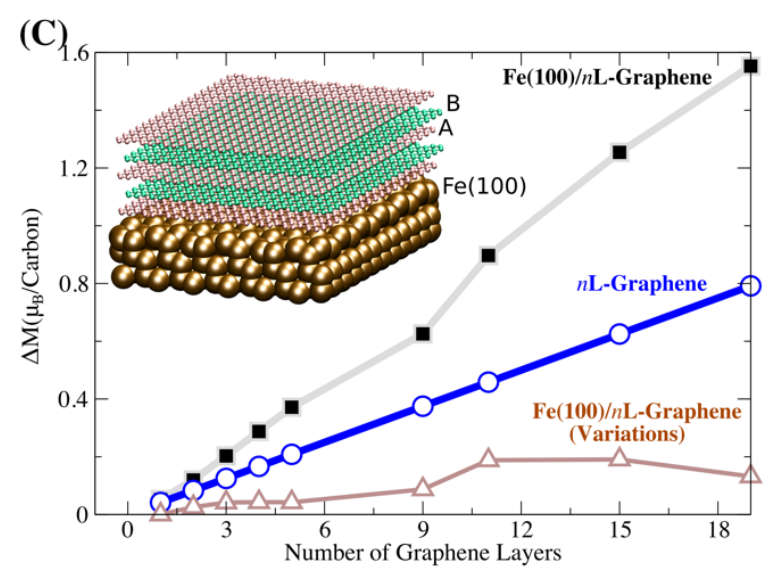

(D)

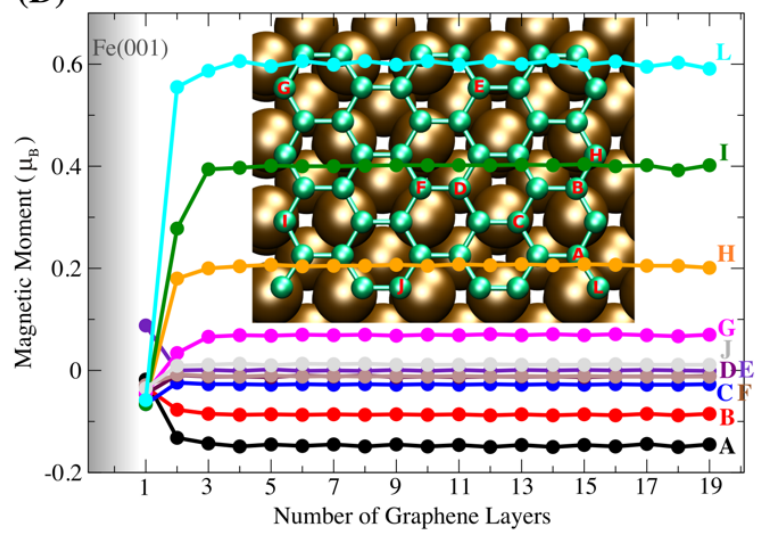

Figure 4 | First-principles simulations of graphene on Fe(100). (A) Schematic of the spin transfer from $\mathrm{Fe}$ to multilayer graphene. The polarized spin states at the interface induce a finite spin-moment in the carbon states. To avoid spurious artefacts from the difference in lattice mismatch between graphene and $\mathrm{Fe}(100)$, graphene layers are modeled in terms of nanoribbons with $\mathrm{H}$-saturated edges as shown in the inset in D. (B) Spin polarization $\xi$ versus energy for different number of graphene layers on Fe (100). $\xi$ is defined in terms of $\xi=n^{U p}-n^{D o w n} / n^{U p}+n^{D o w n}$, where $n^{D o w n}$ and $n^{U p}$ are the density of states of spin up and spin down states, respectively. Spin polarization at different number of layers is highlighted in different colors. Fermi level is set to zero in all curves. Interestingly, the first layer close to the Fe surface is magnetically coupled to $F e$ in a different way than the others. That is, if $\mathrm{Fe}$ is ferromagnetic, $1 \mathrm{~L}$ graphene is antiferromagnetic, and vice-versa. Similar profile of $\xi$ is observed at Fermi level for different number of graphene sheets. (C) Magnetization (Bohr magneton per carbon) versus number of graphene layers for: $F e$ (100)/ $n$ L-graphene systems (gray), isolated $n$ L-graphene sheets (blue) and relative variation of the magnetization per layer for $\mathrm{Fe}(\mathbf{1 0 0}) / \mathrm{nL}$-graphene (faint brown). Inset shows a molecular schematic of the graphene layers on Fe (100) surface at AB stacking. Due to the finite size used to model graphene a net magnetic moment appears at the edges. This magnetic moment increases linearly with the number of sheets (blue curve) but is substantially enhanced when the graphene layers are putted together with $\mathrm{Fe}(100)$. This indicates that the Fe surface has a clear effect on the magnetization of defects/edges once the interface is created. This is in sound agreement with the experiments showed in Figure 2. (D) Local magnetic moment (Bohr per carbon) versus number of graphene layers at some of the carbon atoms onto $\mathrm{Fe}(100)$ substrate. Carbon atoms near defects or 
imperfections (e.g. vacancies) tend to be easily polarized at thicker number of layers, but suffer strong reduction at thinner systems due to competition with charge transfer.

Table 1. The calculated Bohr magneton results from the possible factors which could generate magnetic moment on graphene.

\begin{tabular}{lcc}
\hline Factors & $1^{\text {st }}$ Layer & $2^{\text {nd }}$ Layer \\
\hline Proximity & 0.012 & 0.008 \\
Intercalation & 0.026 & 0.021 \\
Defects & 0.4 & 0.008 (no defects) \\
\hline
\end{tabular}

From our experimental data with the support of theoretical calculations, layer controllable graphene samples grown via CVD could provide controllable magnetic properties. Magnetic properties of graphene samples were measured and confirmed that we have significant changes which resulted from defects through the graphene plane. Moreover, MFM confirm the change of the domain patterns which results from a giant magnetic moment in pure graphene structures.

In conclusion, we have demonstrated a new way to synthesize large area magnetic graphene with controllable magnetic properties. The estimated magnetization of 0.4 Bohr was observed through calculations on a carbon site. The magnetic properties kept after transfer from metal substrates as shown in SI. The controllable magnetic properties of large area carbon nanostructure would be an excellent building block such as spin polarized electron source at Fermi level for future energy efficient spintronic devices. The results pave a new avenue to the light element-based spintronics applications.

\section{Materials and Methods}

Synthesis of Graphene: $\quad$ Graphene was grown on 100- $\mu m$-thick Fe foil (Nilaco, 99.99\% purity) in a hot furnace consisting of a $50 \mathrm{~mm}$ quartz tube. Fe foil was first placed in the center of a horizontal quartz tube. After $\mathrm{H}_{2}$ (100 standard cubic centimeters per unit, sccm) and $\mathrm{Ar}(200$ sccm) were introduced as the carrier gas, and when the furnace temperature reached at $800 \mathrm{~K}$. Fe foil was pre-annealed at $800 \mathrm{~K}$ for $30 \mathrm{~min}$ to remove the native $\mathrm{Fe}$ oxide layer and enlarge the $\mathrm{Fe}$ grains in $\mathrm{H}_{2}$ and $\mathrm{Ar}$ atmosphere. Fe changes its phase above $800 \mathrm{~K}$. Then, $\mathrm{C}_{2} \mathrm{H}_{2}(5 \mathrm{sccm})$ was flowed with the process pressure set to $500 \mathrm{~m}$-Torr during growth. After $\mathrm{C}_{2} \mathrm{H}_{2}$ was exposed, the furnace was cooled to room temperature with $\mathrm{H}_{2}$ and Ar.

Raman Spectroscopy: Raman spectroscopy (Reinshaw, RM1000-Invia) with a laser excitation wavelength of $514 \mathrm{~nm}(2.41 \mathrm{eV})$, a notch filter cut-off frequency of $50 \mathrm{~nm}^{-1}$, and a focus spot size of $5 \mu \mathrm{m}$ was used to measure crystallinity and the number of layers in the graphene films. The few-layer graphene was synthesized for 1, 3, 6, and 9 min, a very strong 2D 
peak at $\sim 2700 \mathrm{~cm}^{-1}$ and a $\mathrm{G}$ peak at $\sim 1580 \mathrm{~cm}^{-1}$ appeared on each Raman spectra, and the D peak at $\sim 1350 \mathrm{~cm}^{-1}$ was observed.

253 Scanning and Transmission Electron Microscopy (SEM and TEM): In order to inspect the exact thickness of the synthesized graphene, high-resolution transmission electron microscopy (HR-TEM, JEOL, JEM-2100F) measurements were performed for estimating the number of 256 layers of graphene.

257 Atomic Force Microscopy/Magnetic Force Microscopy: The scanning probe microscopy 258 (SPM) was performed in non-contact mode using a Bruker-Nano AFM system. The MFM measurements were conducted in a dynamic lift mode with a lift distance of $30 \mathrm{~nm}$. The dynamics were measured in the presence of a magnetic field by sweeping the field range.

Vibrating Sample Magnetometry (VSM): The conventional volume averaging magnetometry measurement were performed using a VSM 7400 of Lake Shore Cryotronics Inc. with a 3.1-T electromagnet. The sample was mounted on a quartz holder. The magnetic moment was measured and averaged. The in-plane crystalline anisotropy is shown in Fig. S5. In this type of crystal structures, the domain is randomly oriented and the size of the domain structures are very large (more than a micron).

First-principles van der Waals ab initio calculations: The calculations reported here are based on ab initio density-function-theory using the SIESTA method ${ }^{27}$ and the VASP code..$^{28,29}$ The generalized gradient approximation ${ }^{30}$ along with the DRSLL ${ }^{31}$ functional was used in both methods, together with a double- $\zeta$ polarized basis set in SIESTA, and a well-converged planewave cutoff of $500 \mathrm{eV}$ in VASP. Projected augmented wave method (PAW) ${ }^{32,33}$ for the latter, and norm-conserving (NC) Troullier-Martins pseudopotentials ${ }^{34}$ for the former, have been used in the description of the bonding environment for $\mathrm{Fe}$ and $\mathrm{C}$. The shape of the NAOs was automatically determined by the algorithms described in S1. The cutoff radii of the different orbitals were obtained using an energy shift of $50 \mathrm{meV}$, which proved to be sufficiently accurate to describe the geometries and the energetics. Atomic coordinates were allowed to relax until the forces on the ions were less than $0.01 \mathrm{eV} / \AA$ under the conjugate gradient algorithm. To model the system studied in the experiments, we created large supercells containing up to 1382 atoms to simulate the interface between multilayer graphene and Fe surfaces. We have initially used three different facets to represent the Fe substrates such as (111), (110), and (100). We have used all three at the monolayer graphene limit, and Fe (100) facet for multilayer graphene, as the high concentration of these facets were found in the Fe foil. To avoid any interactions between supercells in non-periodic direction, a $20 \AA$ was used in all calculations. In addition to this, a cutoff energy of $120 \mathrm{R}_{\mathrm{y}}$ was used to resolve the real-space grid used to calculate the Hartree and exchange-correlation contribution to the total energy. The Brillouin zone was sampled with a $10 \times 10 \times 1$ grid under the Monkhorst-Pack scheme ${ }^{35}$ to perform relaxations with and without van der Waals interactions. A few geometries required a finer grid of $15 \times 15 \times 1$ to fully relax the atomic forces. Energetics were calculated using a converged $25 \times 25 \times 1 \mathrm{k}$-sampling. In addition to this, we used a Fermi-Dirac distribution with an electronic temperature of $k_{B} T=21 \mathrm{meV}$ to resolve the electronic structure.

\section{Author Contribution}


J.H. conceived the idea and designed the experiments. J.H. and C.-C.H. led the experiments (with assistance from Y.K., G.C., A.S.). J.H., L.Y., J.B., C.-C.H. contributed to data analysis and interpretation. J.L., E.J.G.S., and H.-X.Y. performed calculations. J.H. and C.-C.H. wrote the paper and all authors provided feedback.

\section{Acknowledgments}

This work was supported by the National Natural Science Foundation of China under Award number 61674062. J.B. acknowledge financial support from the National Science Foundation (NSF) under Award number 0939514. The work was supported by the U. S. Department of Energy, Office of Basic Energy Sciences, Division of Materials Sciences and Engineering under Contract No. DE-AC02-05CH11231. E.J.G.S. acknowledges the use of computational resources from the UK national high-performance computing service, ARCHER, for which access was obtained via the UKCP consortium and funded by EPSRC (EP/K013564/1), to the UK Materials and Molecular Modelling Hub, which is partially funded by EPSRC (EP/P020194/1 and Department for the Economy (USI 097) for financial support. C.C. H. acknowledges the support by the National Research Foundation of Korea (NRF) funded by the Korean government (Ministry of Science and ICT) (No. 2018R1A5A6075964, No. 2017R1A2B2003928, and No. 2017M3A7B4049173).

\section{References}

1. J. Hong, B. Lambson, S. Dhuey, J. Bokor, Experimental test of Landauer's principle in single-bit operations on nanomagnetic memory bits. Sci. Adv. 2, e1501492 (2016).

2. J. D. Meindl, J. A. Davis, The fundamental limit on binary switching energy for terascale integration. IEEE J. Solid-state Circuits. 35, 1515-1516 (2000).

3. S. A. Wolf, A. Y. Chtchelkanova, D. M. Treger, Spintronics- A retrospective and perspective. IBM J. Res. Develop. 50, 101-110 (2006).

4. C. L. Kane, E. J. Mele, Quantum spin hall effect in graphene. Phys. Rev. Lett. 95, 226801 (2005).

5. W. Han, R. K. Kawakami, Spin relaxation in single-layer and bilayer graphene. Phys. Rev. Lett. 107, 047207 (2011).

6. K. I. Bolotin, K. J. Sikes, Z. Jiang, M. Klima, G. Fudenberg et al., Ultrahigh electron mobility in suspended graphene. Sol. State Comm. 146, 351-359 (2008).

7. N. Tombros, C. Jozsa, M. Popinciuc, H. T. Jonkman, B. J. van Wees, Electronic spin transport and spin precession in single layers at room temperature. Nature 448, 571-574 (2007).

8. M. M. Asmar, S. E. Ulloa, Spin-orbit interaction and isotropic electronic transport in graphene. Phys. Rev. Lett. 112, 136602 (2014).

9. K. S. Burch, D. Mandrus, J.-G. Park, Magnetism in two-dimensional van der Waals materials. Nature 563, 47-52 (2018).

10. R. C. Haddon, Magnetism of carbon allotropes. Nature 378, 249-255 (1995). 
11. O. V. Yazyev, Emergence of magnetism in graphene materials and nanostructures. Rep. Prog. Phys. 73, 056501 (2010).

12. Y.-W. Son, M. L. Cohen, S. G. Louie, Half-metallic graphene nanoribbons. Nature 444, 347 (2006).

13. J. Hong, E. Bekyarova, W. A. de Heer, S. Khizreov, R. C. Haddon, Chemically engineered graphene-based 2D organic molecular magnet. ACS Nano 7, 10011-10022 (2013).

14. M. Sepioni, R. R. Nair, S. Rablen, J. Narayanan, F. Tuna et al., Limits on intrinsic magnetism in graphene. Phys. Rev. Lett. 105, 207205 (2010).

15. J. Hong, E. Bekyarova, W. A. de Heer, R. C. Haddon, S. Khizroev et al., Room-temperature

16. J. Hong, S. Niyogi, E. Bekyarova, M. E. Itkis, P. Ramesh, N. Amos, D. Litvinov, C. Berger, W. A. de Heer, S. Khizroev, R. C. Haddon, Effect of nitrophenyl functionalization on the magnetic properties of epitaxial graphene. Small 7(9) 1175-1180 (2011).

17. S. Niyogi, E. Bekyarova, J. Hong, S. Khizroev, C. Berger, W. A. de Heer, R. C. Haddon, Covalent chemistry for graphene electronics. J. Phy. Chem. Lett. 2, 2487-2498 (2011).

18. N. A. Vinogradov, A. A. Zakharov, V. Kocevski, J. Rusz, K. A. Simonov et al., Formation and structure of graphene waves on Fe (110). Phys. Rev. Lett. 109, 026101 (2012).

19. W. Q. Liu, W. Y. Wang, J. J. Wang, F. Q. Wang, C. Lu, et al., Atomic-scale interfacial magnetism in Fe/Graphene heterojunction. Sci. Rep. 5, 11911 (2015).

20. M. Weber, E. N. Voloshina, K. Horn, Y. S. Dedkov, Electronic structure and magnetic properties of the graphene/Fe/Ni(111) intercalation-like system. Phys. Chem. Chem. Phys. 13, $7534-7539$ (2011).

21. P. Carra, B. T. Thole, M. Altarelli, X. Wang, X-ray circular dichroism and local magnetic fields. Phys. Rev. Lett. 70, 694 (1993).

22. P. Esquinazi, D. Spemann, R. Hohne, Induced magnetic ordering by proton irradiation in graphite. Phys Rev. Lett. (2003).

23. Y. Xue, B. Wu, Y. Guo, L. Huang, L. Jiang, et al., Synthesis of large-area, few-layer graphene on iron foil by chemical vapor deposition. Nano Res. 4(12) 1208 - 1214 (2011).

24. X. Li, W. Cai, J. An, S. Kim, J. Nah et al., Large-area synthesis of high-quality and uniform graphene films on copper foils. Science 324(5932) 1312 - 1314 (2009).

25. K. S. Kim, Y. Zhao, H. Jang, S. Y. Lee, J. M. Kim et al., Large-scale pattern growth of graphene films for stretchable transparent electrodes. Nature 457, 706 - 710 (2009).

26. X. Chen, L. Zhang, S. Chen, Large area CVD growth of graphene. Synth. Met. 210, 95 - 108 (2015).

27. M. S. José et al., The SIESTA method for ab initio order- N materials simulation. J. Phys.: Condens. Matter 14, 2745-2779 (2002).

28. G. Kresse, J. Hafner, Ab initio molecular dynamics for open-shell transition metals. Phys. Rev. B 48, 13115-13118 (1993). 
29. G. Kresse, J. Furthmüller, Efficient iterative schemes for ab initio total-energy calculations using a plane-wave basis set. Phys. Rev. B 54, 11169-11186 (1996).

30. J. P. Perdew, K. Burke, M. Ernzerhof, Generalized gradient approximation made simple. Phys. Rev. Lett. 77, 3865-3868 (1996).

31. M. Dion, H. Rydberg, E. Schroder, D. C. Langreth, B. I. Lundqvist, Van der Waals density functional for general geometries. Phys. Rev. Lett. 92, 246401 (2004).

32. P. E. Blöchl, Projector augmented-wave method. Phys. Rev. B 50, 17953-17979 (1994).

33. G. Kresse, D. Joubert, From ultra-soft pseudopotentials to the projector augmented-wave method. Phys. Rev. B 59, 1758-1775 (1999).

34. N. Troullier, J. L. Martins, Efficient pseudopotentials for plane-wave calculations. Phys. Rev. B 43, 1993-2006 (1991).

35. H. J. Monkhorst, J. D. Pack, Special points for Brillouin-zone integrations. Phys. Rev. B 13, 5188-5192 (1976). 
389 Materials and Methods

\section{Contents}

\section{Supporting Methods}

1. Synthesis of graphene

394 3. Microscopy (AFM/MFM \& SEM \& TEM)

395 4. Vibrating sample magnetometer (VSM) measurements

396 5. X-ray magnetic circular dichroism (XMCD)

397 6. First-principles van der Waals ab initio calculations

398 7. Low energy electron diffraction (LEED) and Auger electron spectroscopy (AES)

\section{Supporting Figures}

S1. The m-H loop of bulk materials

S2. X-ray magnetic circular dichroism (XMCD) measurements

S3. Magnetic simulations for the Fe/Graphene interface

S4. LEED pattern of Fe foil

\section{Materials and Methods:}

1. Synthesis of graphene: Graphene was grown on 100- $\mu m$-thick Fe (foil (Nilaco, $99.99 \%$ purity) in a hot furnace consisting of a $50-\mathrm{mm}$ quartz tube. Fe foil was first 
placed in the center of a horizontal quartz tube. After $\mathrm{H}_{2}$ (100 standard cubic centimeters per unit, $\mathrm{sccm})$ and $\operatorname{Ar}(200 \mathrm{sccm})$ were introduced as the carrier gas, and when the furnace temperature reached at $800 \mathrm{~K}$. Fe foil was pre-annealed at $800 \mathrm{~K}(\mathrm{Fe}$ phase will be changed above $850 \mathrm{~K}$.) for $30 \mathrm{~min}$ to remove the present $\mathrm{Fe}$ oxide layer and enlarge the $\mathrm{Fe}$ grains in $\mathrm{H}_{2}$ and $\mathrm{Ar}$ atmosphere. Then, $\mathrm{C}_{2} \mathrm{H}_{2}(5 \mathrm{sccm})$ was flowed with the process pressure set to $500 \mathrm{~m}$-Torr during growth. After $\mathrm{C}_{2} \mathrm{H}_{2}$ was exposed, the furnace was cooled to room temperature with $\mathrm{H}_{2}$ and Ar.

2. Raman spectroscopy: Raman spectroscopy (Reinshaw, RM1000-Invia) with a laser excitation wavelength of $514 \mathrm{~nm}(2.41 \mathrm{eV})$, a notch filter cut-off frequency of $50 \mathrm{~nm}^{-1}$, and a focus spot size of $5 \mu \mathrm{m}$ was used to measure crystallinity and the number of layers in the graphene films. The few-layer graphene was synthesized for $1,3,6$, and 9 min, a very strong $2 \mathrm{D}$ peak at $\sim 2700 \mathrm{~cm}^{-1}$ and a $\mathrm{G}$ peak at $\sim 1580 \mathrm{~cm}^{-1}$ appeared on each Raman spectra, and the D peak at $\sim 1350 \mathrm{~cm}^{-1}$ was observed.

3. Atomic force microscopy/magnetic force microscopy: The scanning probe microscopy (SPM) was performed in non-contact mode using a Bruker-Nano AFM system. The MFM measurements were conducted in a dynamic lift mode with a lift distance of $30 \mathrm{~nm}$. The dynamic properties were measured in the presence of a magnetic field by sweeping the field range.

4. Vibrating sample magnetometry (VSM): The conventional volume averaging magnetometry measurement were performed using a VSM 7400 of Lake Shore Cryotronics Inc. with a 3.1-T electromagnet. The sample was mounted on a quartz holder. The magnetic moment was measured and averaged. The in-plane crystalline anisotropy is shown in Fig S4. In this type of crystal structures, the domain is randomly oriented and the size of the domain formations are random with larger domains (more than a micron).

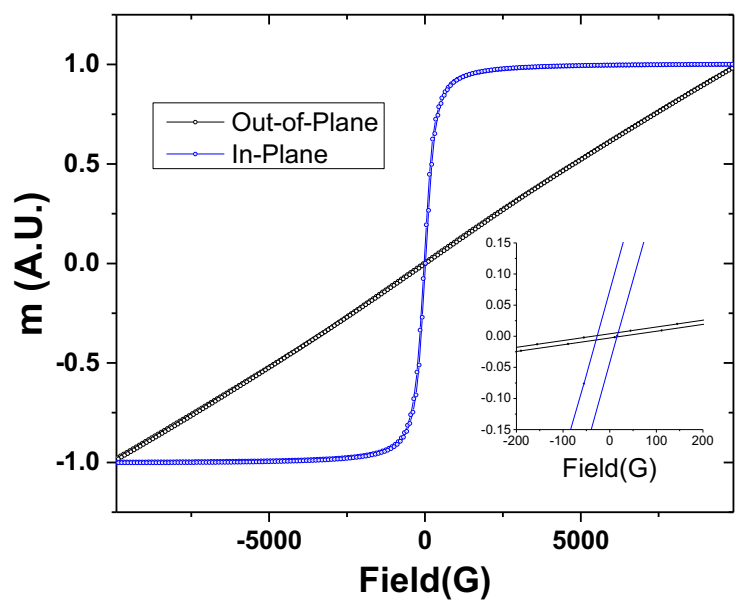

Fig. S1. The m-H loops of bulk Fe foil structures. The easy axis is in-plane and $\mathrm{Fe}(100)$ is the easy axis. The orientation is random, but the majority orientation is $\mathrm{Fe}(100)$. 
5. X-ray spectra: We observed two interesting phenomena: one is that the NEXAFS signal is changing by the incidence of light either normal to the plane or grazing direction which could be very close to in-plane. This would be the significant indication of the nature of $2 \mathrm{D}$ materials. The $C K$-absorption edge XAS spectrum of the graphene/ $\mathrm{Fe}(100)$ foil system in the energy range corresponding to the $1 \mathrm{~s} \rightarrow \pi^{*}$ transition consists of two peaks which can be assigned to transition of $1 s$ electron on the interface states which are result of the hybridization of $C p_{z}$ orbitals of graphene layer and Fe $3 d$ orbitals. The modification and the increasing of the XMCD contrast can be explained by the larger energy splitting between spin-up and spin-down $C$ projected density of states (C-pDOS). XMCD spectra measured at the $C K$-edge can only provide information on the orbital moment. From the negative sign of the XMCD signal, one can conclude that the averaged orbital moment of carbon atoms of the graphene layer is aligned parallel to both, the spin and orbital moments. As shown in Fig S2(B), by increasing the thickness of the graphene layers systematically magnetization increases. A transition of $\mathrm{C} 1 s$ at $C K$-edge results from the change of the plane of the incident X-rays. The transition between $\mathrm{C} 1 s$ exhibit pronounced modifications as the angle changes between the electrical vector of the light and the plane of the sample, reflecting the symmetry of the final state. ${ }^{17}$
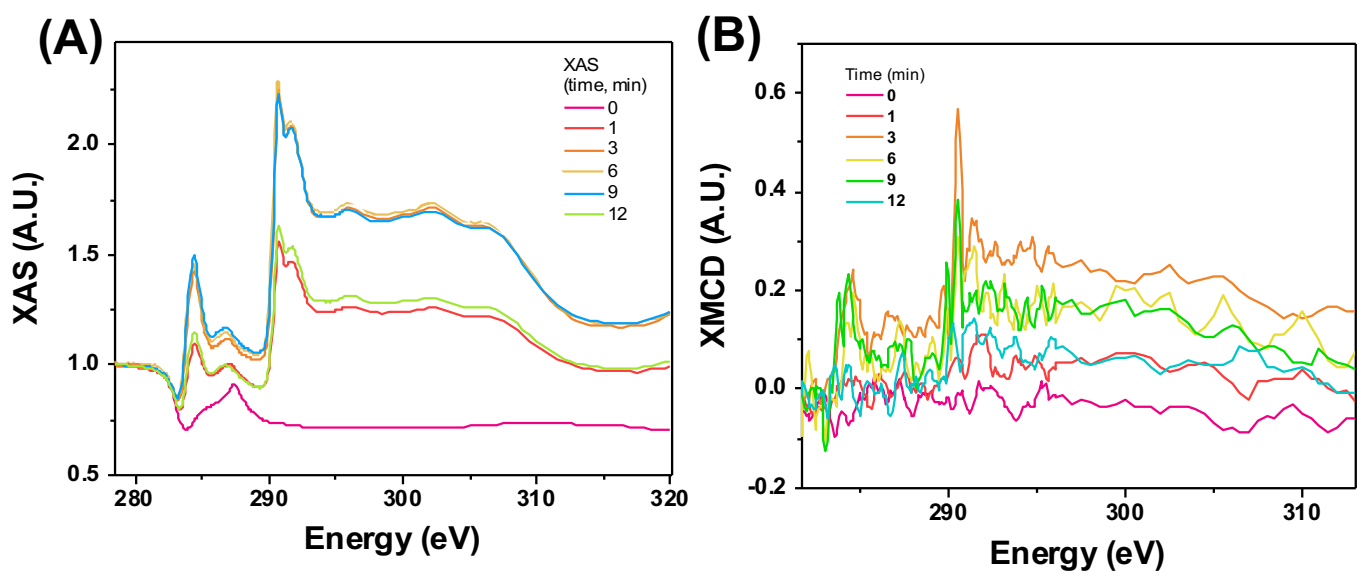

Fig. S2. X-ray magnetic circular dichroism (XMCD) measurements. (A) Layer dependent near edge X-ray absorption fine structure (XAS). (B) The resulting XMCD at $\pi^{*}-$ and $\sigma^{*}$ - edges by increasing the number of layers of graphene.

6. First-principles Van der Waals ab initio calculations: The calculations reported here are based on ab initio density-function-theory using the SIESTA method and the VASP code. The generalized gradient approximation along with the DRSLL functional was used in both methods, together with a double- $\zeta$ polarized basis set in SIESTA, and a well-converged plane-wave cutoff of $500 \mathrm{eV}$ in VASP. Projected augmented wave method (PAW) for the latter, and norm-conserving (NC) TroullierMartins pseudopotentials for the former, have been used in the description of the bonding environment for $\mathrm{Fe}$ and $\mathrm{C}$. The shape of the NAOs was automatically 
(A)

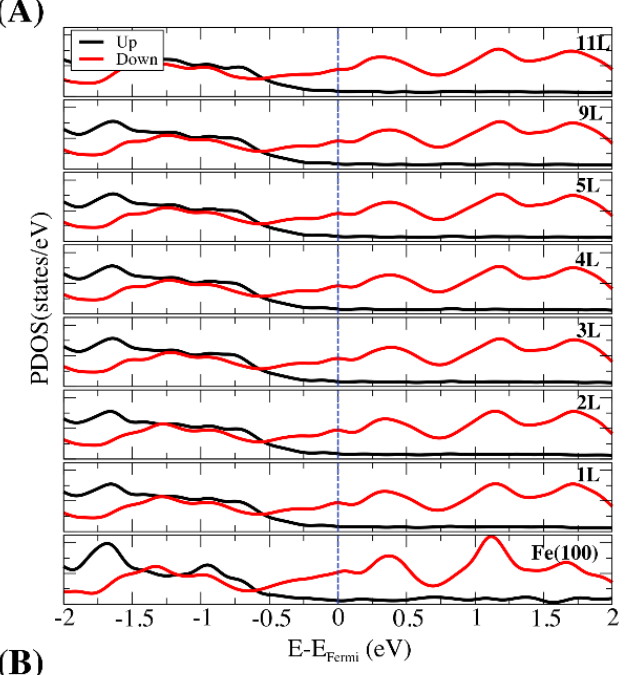

(B)

$2 \mathrm{~L}$
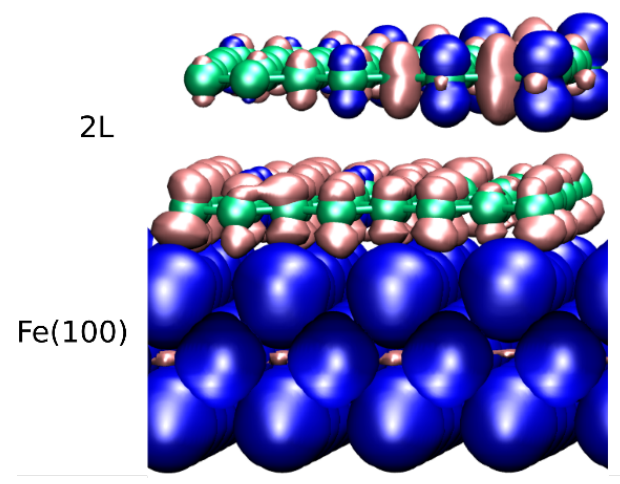

(C)

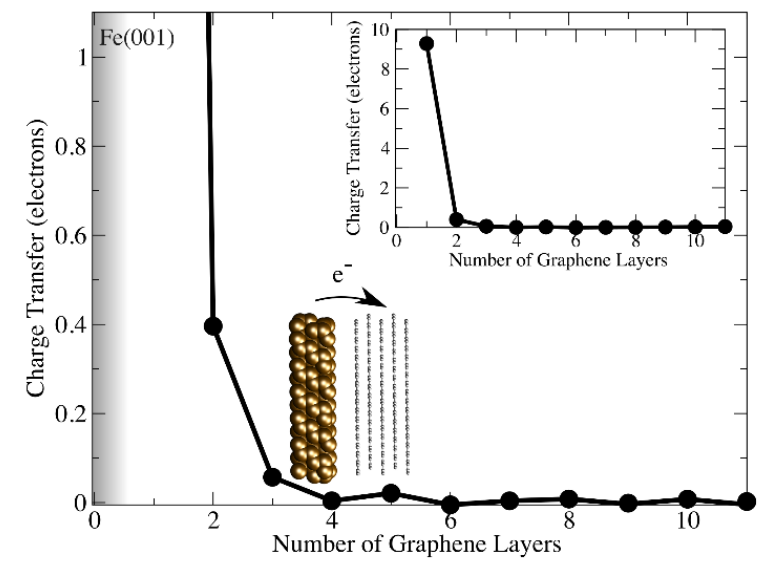

(D)

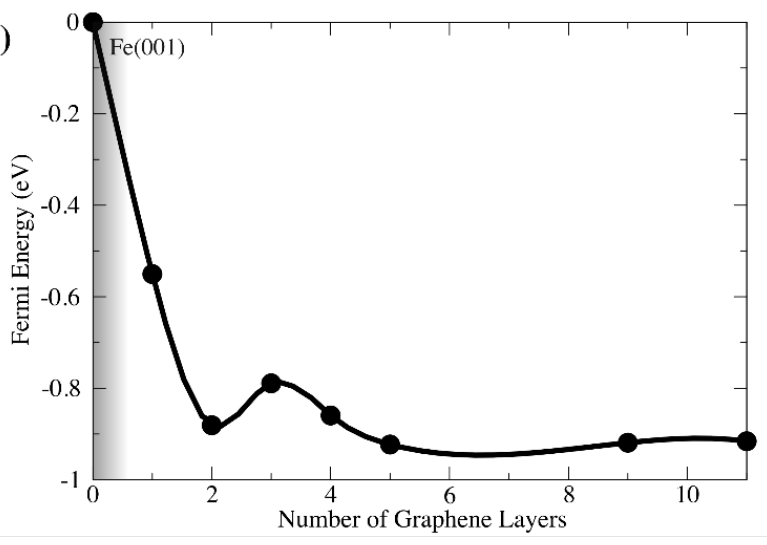

Fig. S3. Magnetic Simulations for the Fe/Graphene interface. (A) Projected density of states (PDOS) on the Fe $3 d$-states for the pristine $\mathrm{Fe}(100)$ slab and at different graphene layers nearby, $n=1,2,3,4,5,9,11$. A substantial amount of spin-polarized electrons are 
observed at the Fermi level with spin-down polarization propagating throughout the layers. Fermi energy is set to zero at all panels. (B) Iso-surface $\left( \pm 0.010\right.$ electrons/Bohr $\left.{ }^{3}\right)$ for the magnetization at the interface between $\mathrm{Fe}(100)$ and $2 \mathrm{~L}$ graphene. Blue and faint pink surfaces show spin-down and spin-up magnetizations, respectively. The strong interaction between the first graphene layer in contact to the Fe surface, changes locally the spin-polarization of the carbon atoms from ferromagnetic (FM) to antiferromagnetic (AFM). The FM order however is recovered at the second graphene layer relative to the Fe surface. (C) Calculated charge-transfer (electrons/per unit cell) into graphene as a function of the number of layers for $\mathrm{Fe}(100) / 11$-layer graphene. The position of the $\mathrm{Fe}(100)$ slab is highlighted at zero layer with the corresponding charge transfer process from Fe (100) to the graphene showed in the scheme. The top inset gives a large-scale perspective along of the charge transfer axis for the first graphene layer in contact to the order of magnitude, relative to the others. The charge also decays exponentially throughout the different carbon sheets, $N$, following a dependence on $\rho \sim \exp (-N / \lambda), \lambda=$ 0.46. (D) Variations of the Fermi energy (eV) versus thickness for $\mathrm{Fe}(100) / 11$-layer graphene. Zero energy is set at the Fermi level of the pristine $\mathrm{Fe}(100)$ slab and all the graphene layers nearby. The oscillatory behavior observed is related with the amount of interfacial charge transfer playing at the different number of layers, which saturates around 5 layers.

\section{Low Energy Electron Diffraction (LEED) \& Spin-polarized low energy electron} microscopy: Through low-energy electron diffraction, the orientation of Fe foil has been investigated. LEED measurement was performed after annealing the sample at 800K within the same UHV system. As shown in Figure S4, Fe(100) orientation has been clearly observed.
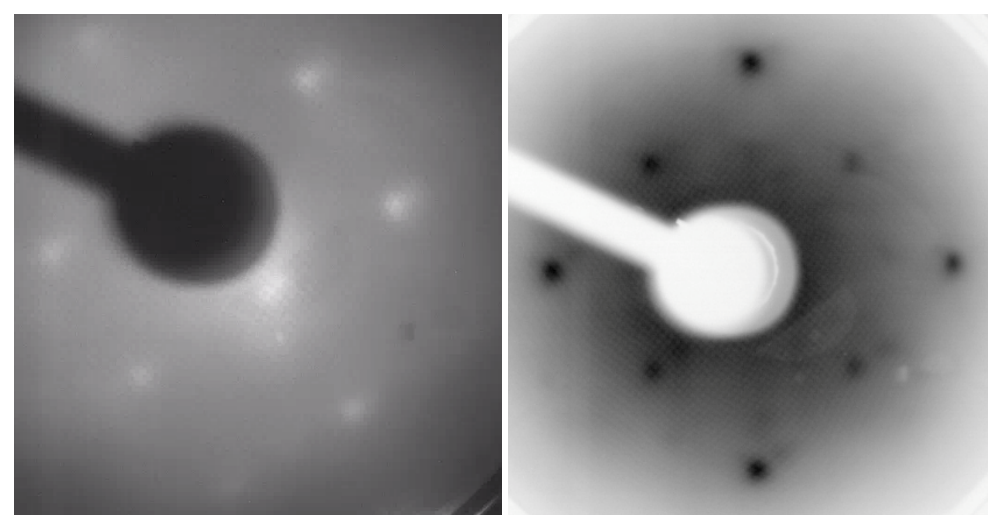

Fig. S4. LEED pattern of Fe foil. The majority crystal orientation is bcc $\mathrm{Fe}(100)$ which shows random magnetic domains ( $\sim$ several $\mu m$-size). The Fe foil has random orientation, but majority could be $\mathrm{Fe}(100)$. $\mathrm{Fe}(100)$ orientation could be easy axis compared to other orientations in the in-plane.

8. Auger Electron Spectroscopy (AES): AES was performed for determining the elemental composition of the several outermost atomic layers of materials. The surface layers often have a composition that is quite different from the bulk material 


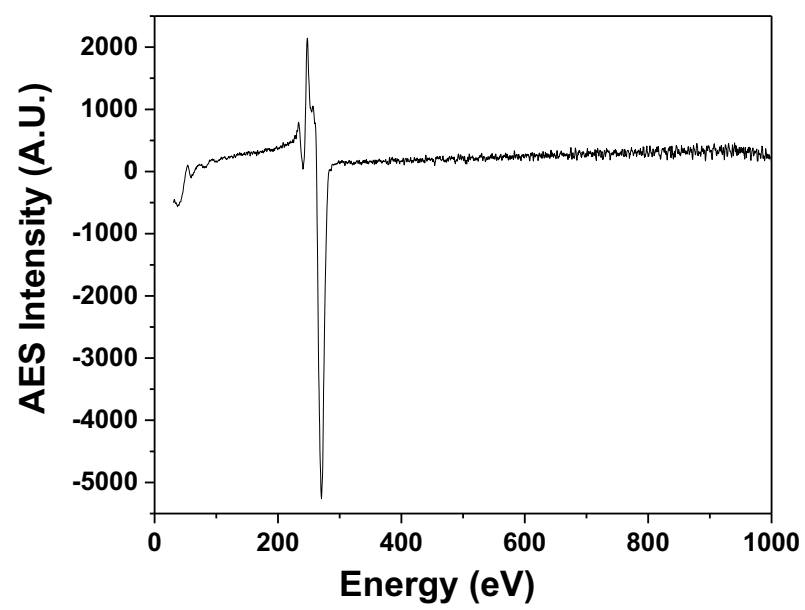

Fig. S5. Auger electron spectroscopy of graphene on Fe sample. The sample shows impurity-free and high quality of graphene with Fe. 